\title{
Pinus aristata, P. balfouriana und P. longaeva, drei bemerkenswerte Kiefernarten aus dem Südwesten der USA
}

\author{
Hubertus Nimsch \& Veit Martin Dörken
}

\begin{abstract}
Pinus aristata, Pinus balfouriana and Pinus longaeva are three similar closely allied species of pine. They are native to the western USA. They can become over 1000 years old. Important distinctions between these species relate to the number of resin channels in the needle leaves. Our own investigations show, however, that the number of resin channels per needle is not a determinant relevant feature, although this is often stated in the literature. The three pine species are presented with their biology, morphology and ecology.
\end{abstract}

\section{Zusammenfassung}

Pinus aristata, Pinus balfouriana und Pinus longaeva sind drei ähnliche Kiefernarten. Sie sind im Westen der USA heimisch und können mit über 1000 Jahren sehr alt werden. Wichtige Unterscheidungsmerkmale beziehen sich auf die Nadeln. Eigene Untersuchungen zeigen, dass aber die Zahl der Harzkanäle pro Nadel kein bestimmungsrelevantes Merkmal ist, obwohl dies in der Literatur oft angegeben wird. Die drei Kiefernarten werden mit ihrer Biologie, Morphologie und Ökologie vorgestellt.

\section{Einleitung}

Der Südwesten der USA gehört zweifelsohne mit zu den Hotspots in der globalen Koniferenvielfalt. So kommen hier zahlreiche Arten der Taxaceae (Eibengewächse), Cupressaceae (Cypressenge-

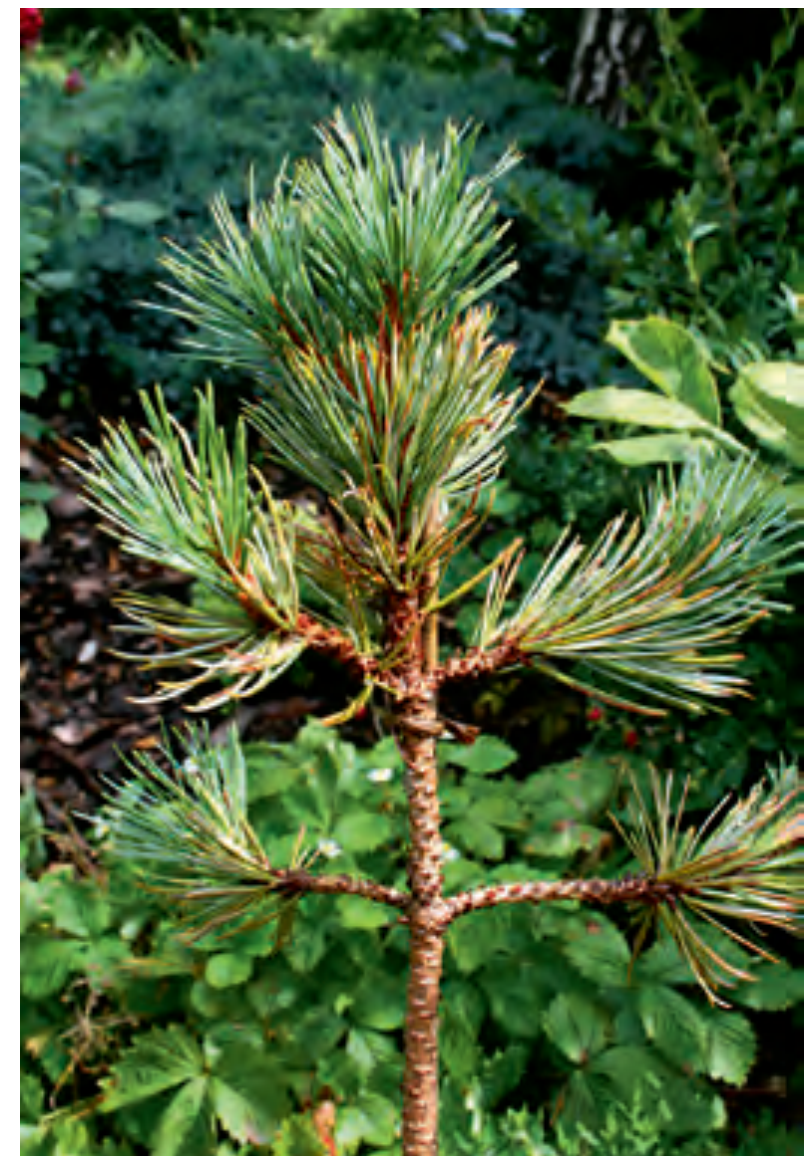

Abb. 1: Jungpflanze von Pinus balfouriana. (Foto: H. Niмsch) wächse) und Pinaceae (Kieferngewächsen) vor. Einige Gattungen wie z. B. Sequoia und Sequoiadendron (Küsten- und Berg-Mammutbaum, beides Cupressaceae) sind dort sogar endemisch. Sie kommen hier zum Teil nur noch in kleinen zersplitterten Reliktarealen vor. Unter den kalifornischen Koniferen gibt es zahlreiche so genannte Champion Trees, die Rekorde unterschiedlicher Natur halten. Der Küsten-Mammutbaum ist mit Wuchshöhen von über $120 \mathrm{~m}$ die höchste Baumart der Erde. Innerhalb der Kieferngewächse sind es besonders die Kiefern, die beeindruckende Rekorde halten. So sind z. B. die Samenzapfen der Coulters-Kiefer (Pinus coulteri) mit einem Gewicht bis $5 \mathrm{~kg}$ die schwersten Koniferenzapfen überhaupt. Die Zapfen der Zucker-Kiefer (Pinus lambertiana) sind mit einer Länge bis $60 \mathrm{~cm}$ die längsten Koniferenzapfen.

Die kalifornischen Koniferen beeindrucken auch in physiologischer Hinsicht. Hier sind dies besonders die Kiefern aus der Sektion Parrya, Subsektion Balfourianae, die auch als Foxtail Pines bezeichnet werden. Zu dieser Gruppe gehören drei nahe verwandte Arten, die alle ein beeindruckendes Alter erreichen können. So ist ein Individuum der Westlichen Grannen-Kiefer (Pinus longaeva), das in den White Mountains im kalifornischen Inyo National Forest steht, mit seinen 5066 Jahren der älteste lebende Baum weltweit. Im Nachfolgenden 
wird die Verbreitung, Morphologie und Ökologie der Foxtail Pines näher vorgestellt.

\section{Zur Systematik der Foxtail Pines}

Die Foxtail Pines werden zur Untergattung Strobus gestellt. Innerhalb dieser werden sie in die Subsektion Balfourianae aus der Sektion Parrya gruppiert. Die Subsektion Balfourianae umfasst nur drei sehr eng verwandte, fünfnadelige Kiefern-Arten: Pinus aristata (Östliche Grannen-Kiefer), Pinus balfouriana (Fuchsschwanz-Kiefer) und Pinus longaeva (Westliche Grannen-Kiefer). Es handelt sich um eine vergleichsweise kleine systematische Gruppe, die keine nähere Verwandtschaft zu den übrigen Arten der Sektion Strobus aufweist (CRitchfield 1977). Die Vorkommen der drei Arten im Südwesten der USA sind räumlich voneinander getrennt. Lange Zeit bestand die Subsektion nur aus zwei Arten, bevor 1970 die Westliche Grannen-Kiefer von der Östlichen Grannen-Kiefer aufgrund deutlicher Unterschiede in der Nadel- und Zapfenmorphologie abgespalten und in die eigenständige Art, Pinus longaeva, überführt wurde (BAILEy 1970). Neben den morpho-anatomischen Merkmalen ist diese Abspaltung auch chemotaxonomisch zu rechtfertigen, da zwischen den beiden Arten deutliche Unterschiede der in den stammbürtigen Harzen und den ätherischen Ölen der Nadelblätter vorhandenen Mono- und Sesquiterpenen bestehen (Zavarin \& Snajberk 1973, Zavarin et al. 1976, 1982, SснÜtт \& Lang 2004b). Versuche, Pinus aristata mit Pinus longaeva zu kreuzen, blieben bislang relativ erfolglos (CRITCHFIELD 1977). Dies ist ein weiteres Argument dafür, dass die taxonomische Splittung in zwei eigenständige Arten durchaus zu rechtfertigen ist. Allen drei Arten ist gemeinsam, dass ihre Nadeln zu fünft pro Kurztrieb stehen, der Nadelrand keine Zähne aufweist und der Samenflügel leicht ablösbar ist (Critchfield 1977).

\section{Die drei Foxtail Pines}

Alle drei Arten der Foxtail Pines sind Bäume, die in Gebirgslagen an der Waldgrenze anzutreffen sind. Ihre ökologische Funktion ist für den Erhalt der Vegetation an der Waldgrenze von enormer Bedeutung. Dass bis heute die zum Teil mehre- re Jahrtausende alten Bäume erhalten geblieben sind, ist wohl nur der Tatsache geschuldet, dass eine wirtschaftliche Bedeutung der Wälder an der Waldgrenze dort nie aktuell war. Zudem hat der für alle fünfnadeligen Pinus-Arten sehr gefährliche Blasenrostpilz Cronartium ribicola am Naturstandort keine Bedeutung, da hier für die Existenz des Pilzes lebensfeindliche Bedingungen (unter anderem Dürre, Hitze, Kälte, Schnee- und Eisbelastungen, hohe UV-Belastungen) herrschen. Dagegen ist die Gefährdung durch den Pilz an kultivierten Exemplaren in niederen Gebieten zum Teil gravierend, auch fernab des Naturstandortes in Deutschland. So wurden z. B. im Arboretum Freiburg-Günterstal an allen Arten der Subsektion Balfourianae Pilzinfektionen durch Cronartium ribicola festgestellt. Demnach ist das Fehlen eines Befalls durch Cronartium ribicola nur auf die am Naturstandort vorherrschenden Bedingungen zurückzuführen und nicht etwa auf eine genetisch fixierte, endogene Widerstandsfähigkeit.

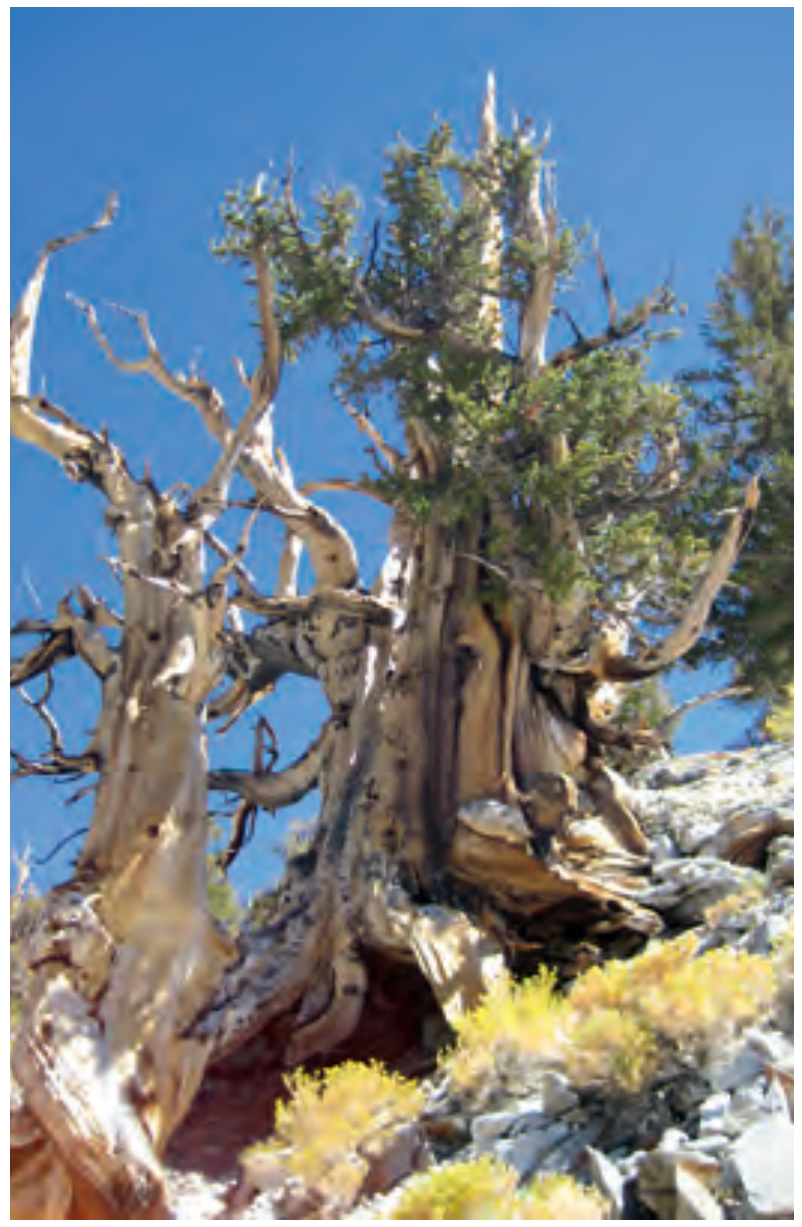

Abb. 2: Altes wettergegerbtes Exemplar von Pinus longaeva am Naturstandort. (Foto: H. NiMSCH) 


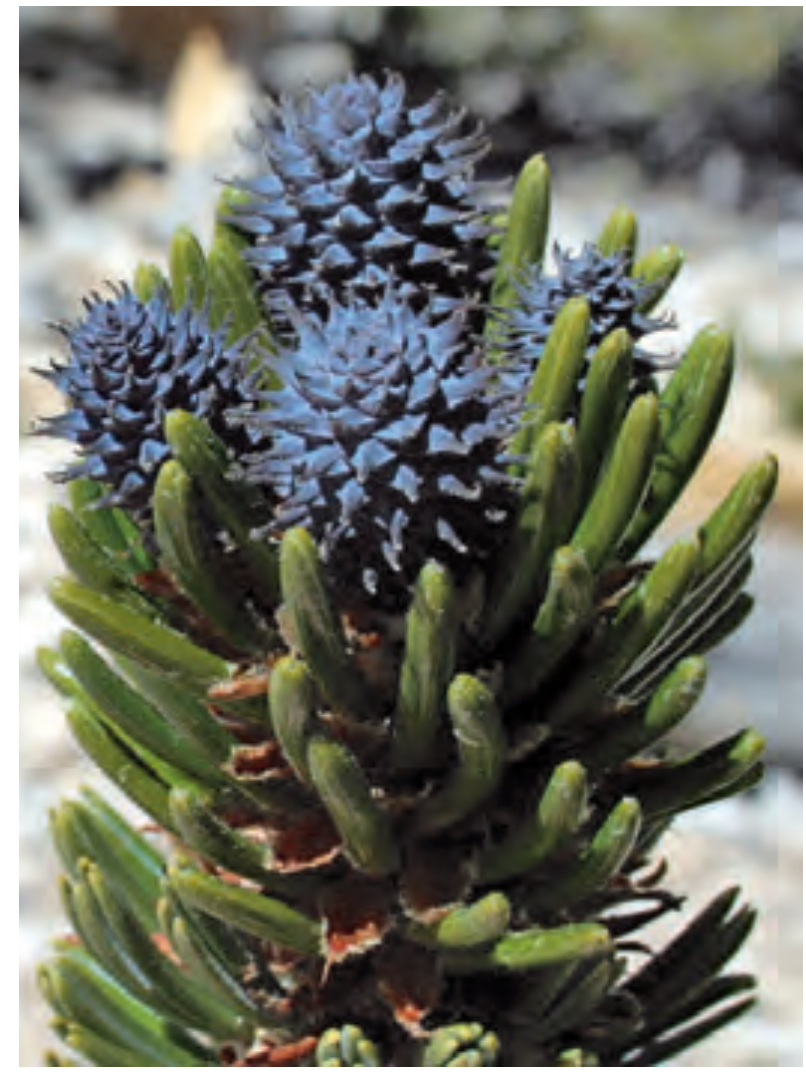

Abb. 3: Zweig mit jungen Samenzapfen von Pinus longaeva (Foto: H. Nimsch)

\subsection{Pinus balfouriana A. MurraY, Fuchsschwanz-Kiefer}

Von Pinus balfouriana werden zwei nur in Kalifornien beheimate Unterarten unterschieden. Die Teilareale sind etwa $700 \mathrm{~km}$ voneinander entfernt. Das nördliche Teilareal mit Pinus balfouriana ssp. balfouriana befindet sich im nordwestlichen Kalifornien und reicht von den Siskiyou- und Yolla Bolly Mountains im Süden bis zu den Salmon-, Scott- und Marble Mountains im Norden (um $40^{\circ}$ n. Br.). Dort kommt diese Unterart in Höhenlagen zwischen 1500-1800 m über NN vor (SchüTt \& LANG 2004b). Das südliche Verbreitungsgebiet mit Pinus balfouriana ssp. austrina befindet sich an den Osthängen der südlichen Sierra Nevada (35-38 n. Br.) im Kings Canyon- und Sequoia National Park, sowie an weiteren kleineren Standorten, darunter im Onion Valley. Hier werden Standorte in über 3000 m über NN besiedelt. In den Inyo Mountains nähern sich die Areale von Pinus balfouriana und Pinus longaeva bis auf ca. 20 Meilen an (Schütт \& LANG 2004a).
Pinus balfouriana wächst unter extremen Klima- und Standortsbedingungen und markiert die kalifornische Baumgrenze in Regionen, in denen die jährliche Vegetationszeit zum Teil unter zwei Monaten liegt (SchütT \& LANG 2004a). Die Art verträgt anhaltende Kälte, hohe Schneedecken, permanenten Sturm, große Hitze und Dürre. Die Hauptwasserversorgung erfolgt an diesen Standorten durch Schmelzwasser (ScHüTT \& LANG 2004a). Hier bildet die Art keine flächendeckenden geschlossenen Bestände aus. Die Bäume stehen oft einzeln und weit voneinander entfernt. Sie bilden aber immer, auch unter schwierigen Wuchsbedingungen, aufrechte Stämme mit oft verdickten Stammbasen, wobei die Stamm- und Kronenform durch die vorherrschenden rauen klimatischen Verhältnisse sehr unregelmäßig gestaltet sind. Bemerkenswert ist die Tatsache, dass die Art auch an der Baumgrenze immer noch stammbildend ist und nicht strauchig wächst. In den mittleren und den höchsten Gebirgslagen wächst Pinus balfouriana in sehr lockeren Reinbeständen oder häufiger in weit voneinander stehenden Einzelbäumen oder Gruppen. Nur an der unteren Verbreitungsgrenze tritt die Art in Mischung mit Pinus albicaulis, Abies magnifica, Pinus contorta, Tsuga mertensiana, Juniperus occidentalis und Juniperus osteosperma auf (SснÜTT \& LANG 2004a) bzw. wird von diesen Arten verdrängt.

Im Vergleich zu Pinus aristata und P. longaeva ist das Höchstalter von P. balfouriana mit 1000 Jahren recht gering (SchüTt \& LANG 2004a). Selbst im Alter werden Wuchshöhen von 20-25 m kaum überschritten. Die jungen Triebe sind dunkelbraun und unregelmäßig behaart. Das harte und harzreiche Holz von Pinus balfouriana ist nicht von $P$. longaeva und $P$. aristata zu unterscheiden (Sснütт \& Lang 2004b). Je Kurztrieb werden 5 relativ steife Nadelblätter hervorgebracht, welche dem Zweig dicht anliegen. Die Nadelspitze ist zugespitzt, leicht gebogen und zeigt zum Langtrieb hin. Die Gesamtnadelblattlänge beträgt lediglich nur $2-4 \mathrm{~cm}$, damit weist $P$. balfouriana die kleinsten Nadelblätter aller fünfnadeligen Kiefern auf (SснÜтt \& LANg 2004a). Dies kann als Anpassung an die am Naturstandort herrschende Dürre, Nährstoffarmut und kurze Vegetations- 
periode angesehen werden. Die abaxiale Seite der Nadel ist tiefgrün, die Innenseiten graugrün mit weißen Stomatabändern (Sснüтt \& LANG 2004a). Die zerriebenen Nadeln duften angenehm aromatisch gegenüber denen von $P$. aristata, welche zerrieben einen doch eher strengen Duft aufweisen. Die Nadeln können 10-20 Jahre alt werden. Die drei Arten der Subsektion Balfourianae blühen etwa gleichzeitig im Juli und August (Sснӥтt \& LANg 2004a), also später als alle anderen nordamerikanischen Kiefernarten (CRITCHFIELD 1977). Der Hauptblütezeitpunkt liegt dabei meist in der letzten Juliwoche. Die Pollenzapfen von $P$. balfouriana sind gelb. Die Samenzapfen sind zum Zeitpunkt der Bestäubung und in der nachfolgenden Entwicklung zunächst stumpf schwarzblau, bevor sie sich in ein Rotbraun verfärben. Die reifen Samenzapfen werden 6-14 cm lang und 4-5 cm breit. Die schmalen, viereckigen Samenschuppen haben ein dickes quergekieltes Zapfenschild. Diesem sitzt ein im Vergleich zu P. aristata winziger, gekrümmter Dorn auf. Die geflügelten, 5-7 mm langen Samen sind matt purpurrot und leicht gefleckt. Der Samenflügel ist $20-25 \mathrm{~mm}$ lang, selten sogar noch etwas länger.

Für die Ausbreitung der Samen aller drei Arten der Subsektion Balfourianae ist der Kiefern-Häher (Nucifraga columbiana) verantwortlich. Dieser vergräbt die reifen Samen in bis zu $30 \mathrm{~cm}$ tiefen Löchern. Dabei werden die Samen nicht einzeln, sondern immer zu mehreren zusammen in einem Loch hinterlegt und verscharrt, was eine wichtige Grundvoraussetzung für eine erfolgreiche Naturverjüngung im Bestand ist. Durch das Verstecken mehrerer Samen beieinander kommt es auch zum dichten Keimen der Samen. Diese dicht stehenden Sämlinge wachsen dann entweder zu einzelnen, sehr dicht stehenden Baumgruppen heran oder die Sämlinge verwachsen im Alter vollständig zu einem einzelnen Baum, in diesem Falle dann mit sehr dickem Stamm (Sснӥтt \& LANG 2004b).

\subsection{Pinus longaeva D. K. BAILEY, Westliche Grannen-Kiefer}

Die natürliche Verbreitung von $P$. longaeva erstreckt sich von den im Westen gelegenen White Mountains (Ost-Kalifornien) weiter nach Südos-

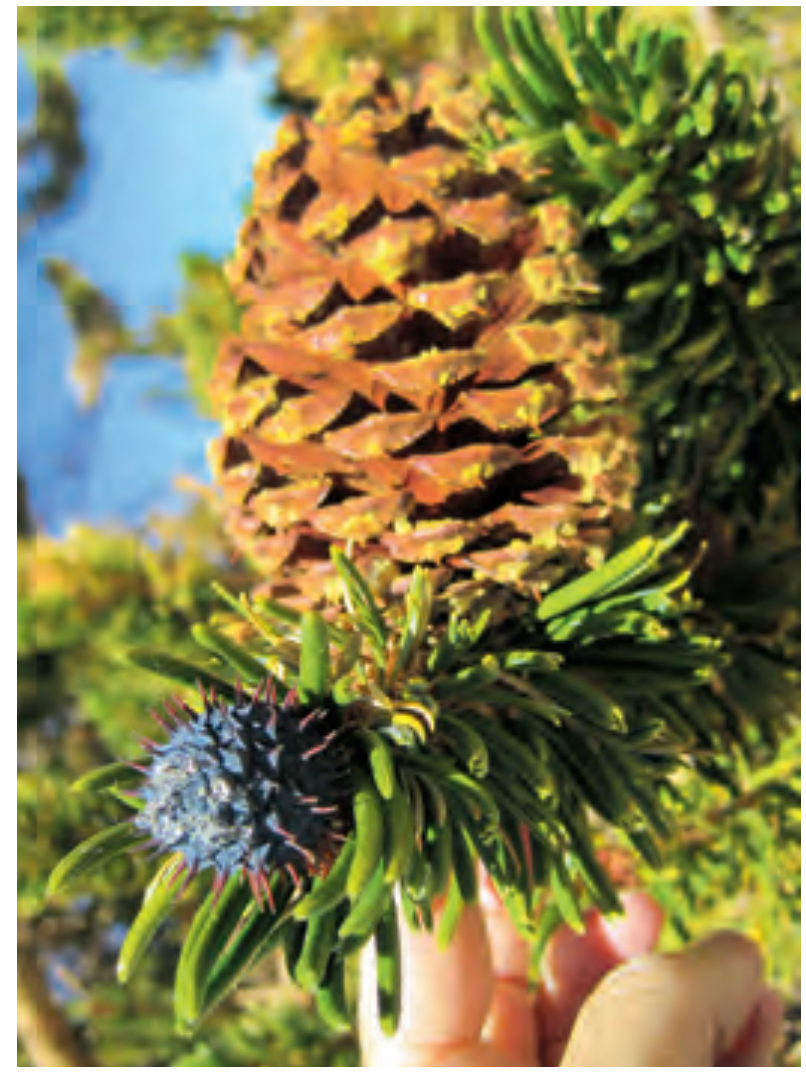

Abb. 4: Junger und ausgewachsener Zapfen von Pinus longaeva. (Foto: H. Nimsch)

ten und schließt große Teile des Great Basins in den Bundestaaten Utah (hier die Nordost-Grenze der Sowers Canyon) und Nevada (White Pine County im Osten und Clark County im Süden) mit ein (Schütt \& Lang 2004b, Eckenwalder 2009, FARJON 2010). Hier kommt die Art aber nicht flächendeckend, sondern in vielen kleinen Teilpopulationen vor (Schütt \& LANG 2004b). Pinus longaeva wächst in lockeren Reinbeständen an der natürlichen Baumgrenze in 3000 bis $4000 \mathrm{~m}$ über NN. Die Temperaturen liegen dort in den Wintermonaten von November bis April immer unter $0^{\circ} \mathrm{C}$ und in den wärmsten Monaten Juli und August auch kaum über $10^{\circ} \mathrm{C}$. Das bedeutet eine sehr kurze Vegetationszeit und erklärt das ausgesprochen langsame Wachstum. Die Niederschläge von etwa $300 \mathrm{~mm}$ fallen hauptsächlich als Schnee (Sснӥтt \& LANg 2004b). Aufgrund der geringen Niederschläge am Naturstandort beschreibt FARJOn (2010) die Art daher auch als Xerophyten. Regelmäßige Winterstürme prägen diese Extremstandorte. Nur in den Randbereichen wie z. B. in den tieferen Gebirgslagen, kommt P. longaeva mit 
P. flexilis vergesellschaftet vor (SchüTt \& LANG 2004b). Durch das deutlich schnellere Wachstum von $P$. flexilis wird $P$. longaeva massiv bedrängt und an einer weiteren Ausbreitung gehindert.

Das Vorkommen von Pinus longaeva ist hauptsächlich auf das helle Dolomit-Gestein und dessen Verwitterungsprodukte begrenzt. Dieses reflektiert $25 \%$ des Sonnenlichtes, so dass eine geringere Bodentemperatur als auf dunklen Böden erzielt wird, wodurch das Wachstum begrenzt ist (THIEL 1989). Besonders die auf diesen Dolomit-Standorten wachsenden Individuen haben eine besonders lange Lebensdauer ihrer Nadeln (Thiel 1989). Die Erosion auf stark geneigten Gebirgsstandorten ist natürlich größer im Vergleich zu nur leicht geneigten Hängen. Dies bedeutet, dass im Laufe von tausenden von Jahren die Erosion die Wurzeln freilegt und ein teilweises Absterben dieser zur Folge hat. Die dann verbleibenden schmalen Rindenstreifen sind in der Lage, ein Weiterleben des Baumes über viele Jahre zu ermöglichen (Thiel 1989). Bedingt durch einen sehr lockeren, weitständigen Standraum ohne Humusauflage und brennbaren Unterwuchs kann ein Feuer, das durch Blitzschlag verursacht wird, nur wenig Schaden anrichten. Auf den dichter bewachsenen Nordhängen der Gebirge kann Feuer hingegen vergleichsweise größeren Schaden verursachen. Für die Dendrochronologie ist die Widerstandsfähigkeit der lebenden Bäume und ihres toten Holzes gegen Fäulnis von besonderer Bedeutung. An über 5000 Jahre alten, lebenden Bäumen in den White Mountains und zusammen mit nicht verrottetem toten Holz längst abgestorbener Bäume ist es gelungen, eine lückenlose Jahrringchronologie von $P$. longaeva aufzustellen. Diese reicht bis 6700 Jahre vor Chr., also über 8700 Jahre vor heute zurück. Das ist ein einmaliger Altersrekord, der auch zur Überprüfung von ${ }^{14} \mathrm{C}$-Methoden zur Altersbestimmung herangezogen wurde (Thiel 1989).

Pinus longaeva erreicht meist nur eine Höhe von 5-10 m. Nur in Ausnahmefällen werden $20 \mathrm{~m}$ Höhe erreicht. Die überwiegend $2,5-3,5 \mathrm{~cm}$

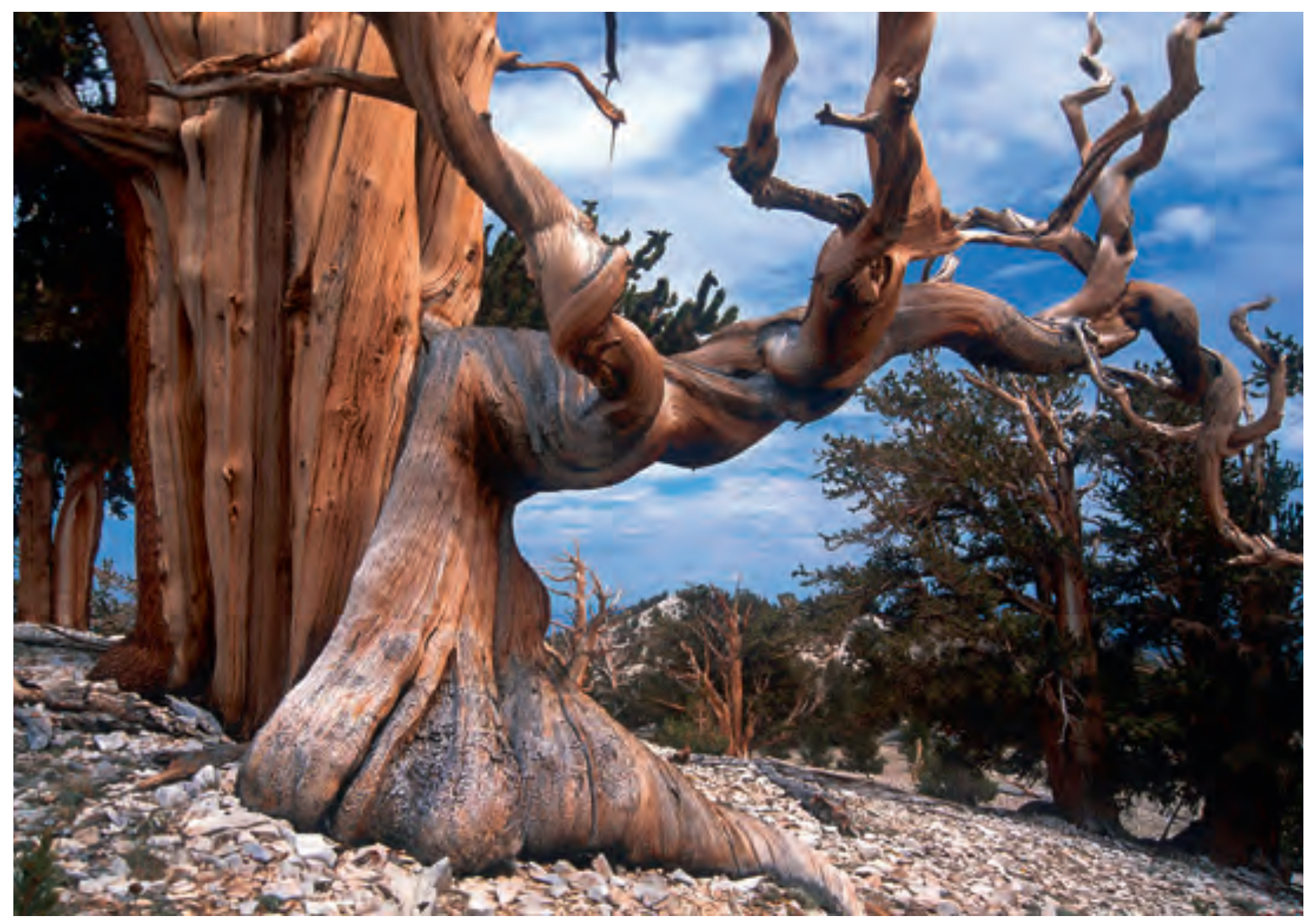

Abb. 5: Sehr altes knorriges Exemplar von Pinus aristata am Naturstandort (Foto: H. Nimsch) 


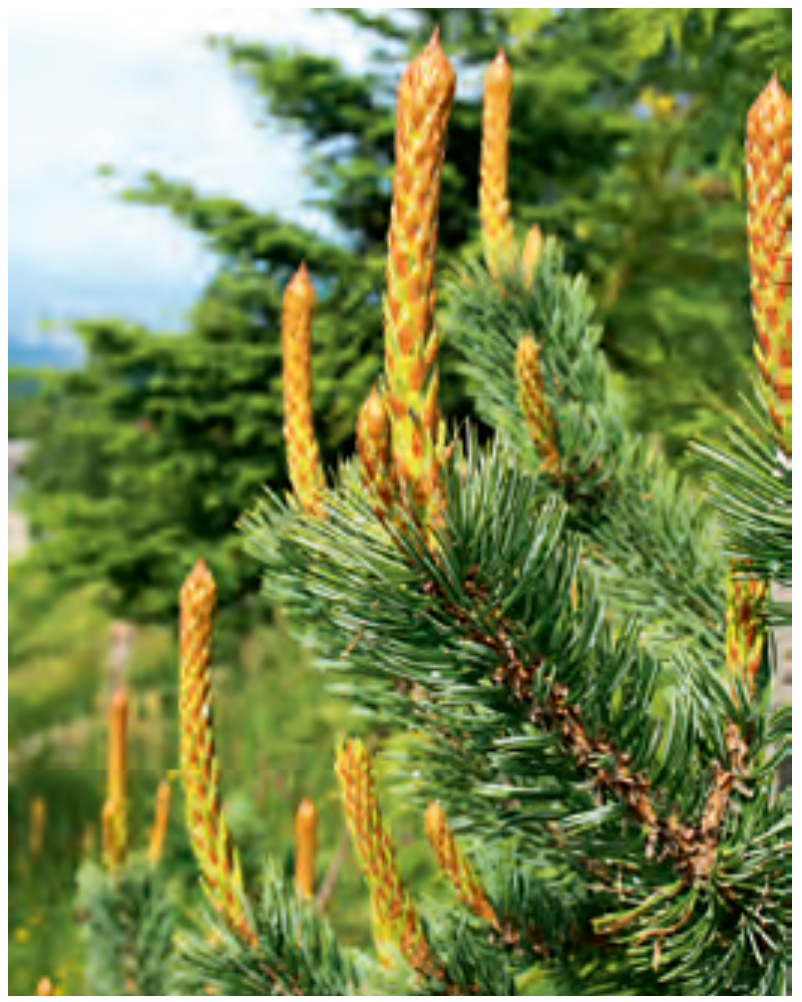

Abb. 6: Pinus aristata in St. Ulrich. (Foto: H. Nimsch)

langen Nadeln stehen meist zu fünft, seltener zu viert, an einem Kurztrieb beisammen ( \& LANG 2004b). Die Hüllblätter am Kurztrieb fallen im ersten Jahr ab. Die grünen Nadelblätter liegen dem Zweig dicht an, sind relativ steif und haben eine stumpfe Spitze (Unterschied zu P. balfouriana). Die Nadeln können ein Alter von 30 Jahren und mehr erreichen (SснÜтT \& LANG 2004b), eine unter Koniferen einzigartige Lebensdauer. Diese lange Lebensdauer der $\mathrm{Na}$ deln ist wahrscheinlich unter anderem darin zu begründen, dass die Anteile des Phloems im Blattleitbündel jährlich neugebildet werden (SснÜтT \& Lang 2004b, Eckenwalder 2009). Auffällig ist die Dicke der Blattkutikula. Diese ist ungefähr doppelt so dick wie bei anderen Koniferen der Baumgrenze (Sснӥтt \& LANG 2004b). Auch wenn sich $P$. longaeva und $P$. aristata in der Benadelung recht ähnlich sind, kann man sie anhand zweier Nadelmerkmale sicher unterscheiden. Pinus aristata: Die Nadeln haben immer deutliche weiße Harzflocken, die Harzkanalscheide ist dünnwandig und parenchymatisch. Pinus longaeva: Die Nadeln haben wenige oder gar keine weiße Harzflocken, die Harzkanalscheide ist dickwandig und massiv sklerenchymatisch.

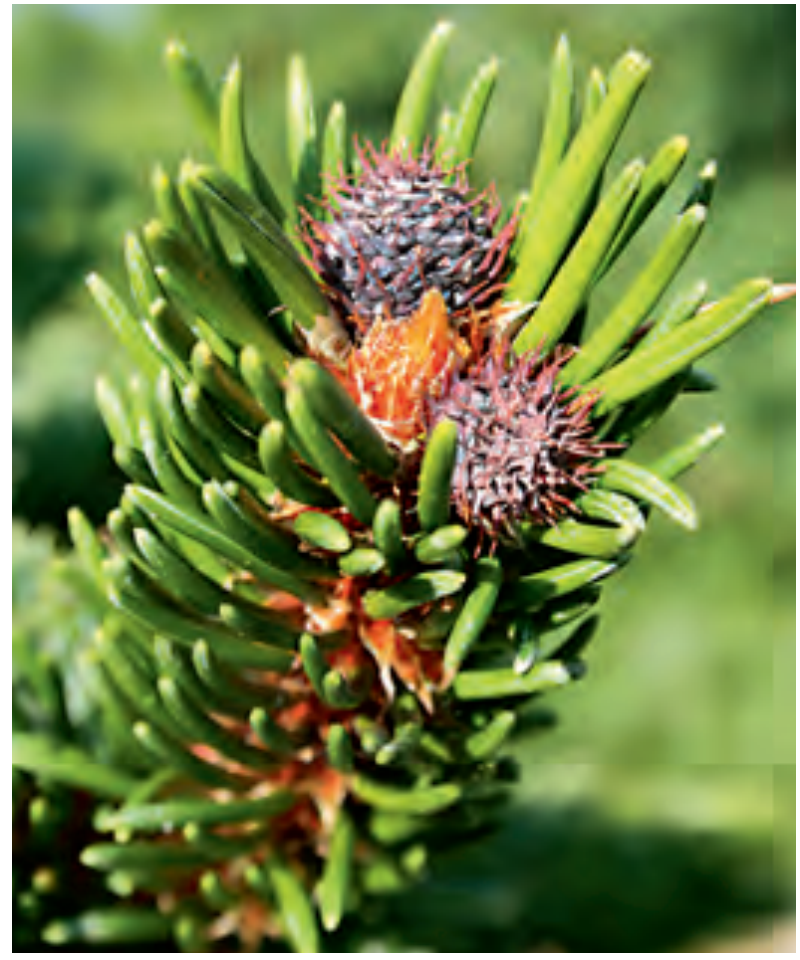

Abb. 7: Junge Samenzapfen an einem in St. Ulrich kultivierten Exemplar von Pinus aristata. (Foto: $\mathrm{H}$. Nimsch)

In der Literatur wird die Anzahl der Harzkanäle je Blatt häufig als ein wichtiges Bestimmungsmerkmal herangezogen. So wird für $P$. aristata ein Harzkanal je Blatt genannt, für $P$. longaeva dagegen zwei (z. B. Schütt \& LANG 2004b, ECKENWALDER 2009). Eigene Untersuchungen haben aber gezeigt (vgl. Tab. 1), dass die Anzahl an Harzkanälen je Blatt kein geeignetes Bestimmungsmerkmal darstellt, da sowohl an einem Kurztrieb Nadeln mit entweder einem oder zwei, manchmal sogar keinem Harzkanal zu finden sind (Abb. 5), wie dies nicht nur für $P$. aristata und $P$. longaeva, sondern auch für $P$. balfouriana zutrifft.

Die Pollenzapfen sind rötlich (bei $P$. aristata und $P$. balfouriana gelb). Die kaum harzigen Samenzapfen sind zur Zeit der Bestäubung auffällig blau, danach verfärben sie sich in ein Purpurrot und zur Reifezeit sind sie rötlich braun. Der reife Samenzapfen ist 5,5-8,5 cm lang. Die Zapfenbildung erfolgt bis ins hohe Alter hinein. So sind selbst auch noch an über 4000 Jahre alten Bäumen junge Samenzapfen zu finden. Die braunen Samen sind 5-8 mm lang und rötlich gefleckt, der Samenflügel ist verglichen mit $P$. balfouriana deutlich kleiner und nur etwa 6-15 mm lang. 


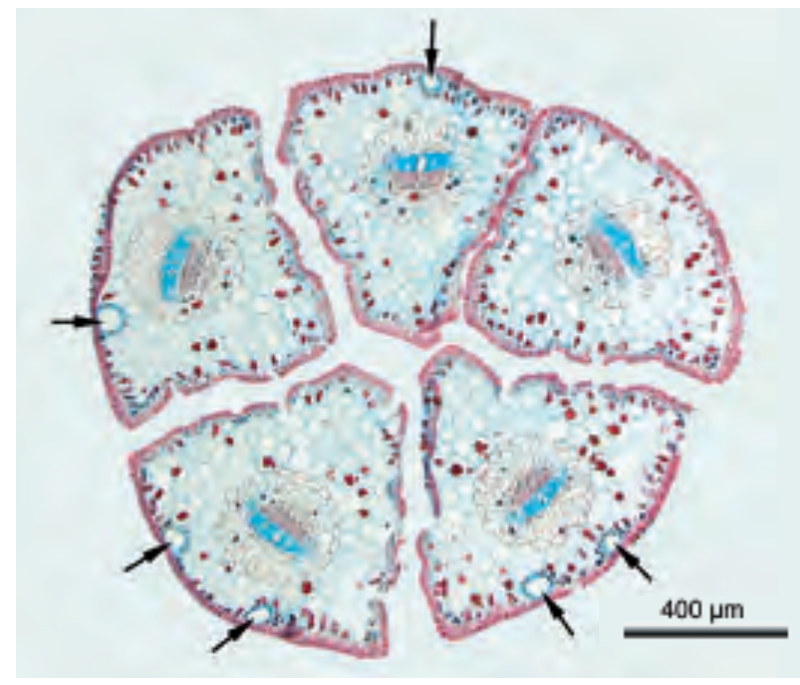

Abb. 8: Pinus aristata, Querschnitt durch einen fünfnadeligen Kurztrieb. Zwei Nadeln mit zwei Harzkanälen (Pfeile), zwei Nadeln mit jeweils einem Harzkanal (Pfeil), eine Nadel ohne Harzkanal. (Foto: V. M. Dörken)

Das hohe Alter, das diese Art erzielen kann, ist auf mehrere Faktoren zurückzuführen. Das Klima in den White Mountains ist kühl und arid. Die Niederschläge variieren von Jahr zu Jahr, sie liegen zwischen 200 und $500 \mathrm{~mm}$ je Jahr. Etwa $80 \%$ entfallen auf Schneeanteile, so dass auf den Sommer nur etwa $100 \mathrm{~mm}$ kommen (Thiel 1989). Die Sommermonate sind in der dünnen Atmosphäre von über $3000 \mathrm{~m}$ sehr heiß. In der sehr trockenen Höhenluft und der winterlichen Kälte können sich Fäulnispilze nicht entwickeln. Auch der für die fünfnadeligen Pinus-Arten sehr gefährliche Blasenrostpilz, Cronartium ribicola, kann in diesen extremen Hochlagen nicht überleben. Neben diesen Faktoren spielt aber auch die Telomerlänge und die Telomerase-Aktivität eine entscheidende Rolle an der Langlebigkeit dieser Art. So haben neuere genetische Untersuchungen gezeigt, dass der Anstieg sowohl in der Länge der Chromosomenendstücke (Telomere) als auch in der Aktivität der Telomerase (ein Enzym,

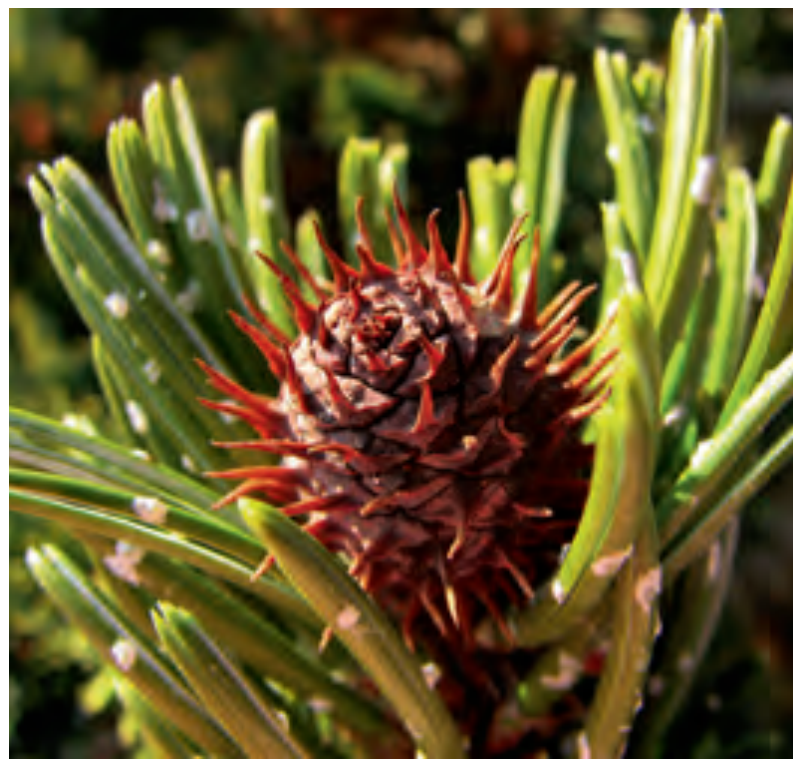

Abb. 9: Junger Samenzapfen an einer im Botanischen Garten Bochum kultivierten Pinus aristata. (Foto: V. M. Dörken)

das diese Endstücke wiederherstellt) bei $P$. longaeva zu einer deutlichen Erhöhung der Langlebigkeit beigetragen hat (Flanary \& KLETETSChKa 2005).

\subsection{Pinus aristata Engelm., Östliche Grannen-Kiefer}

Pinus aristata kommt in den Rocky Mountains von Zentral-Colorado bis ins nördliche New Mexiko, Utah, Nevada und zu den San Francisco Peaks in Arizona vor. Zudem ist sie in den White Mountains und Panamint Mountains in Südost-Kalifornien anzutreffen (Mirov 1967; Eckenwalder 2010). Dort liegt das Verbreitungsgebiet auf extrem trockenen, im Winter genau so extrem kalten Standorten in Höhenlagen zwischen 2750-3650 m über NN an der Waldgrenze (WeIss 2012). Ihre ökologische Bedeutung ist zum Erhalt der Vegetation an den Extremstandorten mit Schnee, Sturm und sommerlicher Hitze sehr wichtig. In diesen Regionen ist die Vegeta-

\begin{tabular}{llllll}
\hline \multirow{2}{*}{ Art } & \multicolumn{3}{l}{ Anzahl an Harzkanälen [n= 25] } & Harzkanalscheide & Harzflocken \\
\cline { 2 - 5 } & $\mathbf{0}$ & $\mathbf{1}$ & $\mathbf{2}$ & & \\
\hline Pinus aristata & 6 & 14 & 5 & parenchymatisch & zahlreich \\
\hline Pinus balfouriana & 2 & 10 & 13 & extrem & fehlend \\
\hline Pinus longaeva & 3 & 5 & 17 & sklerenchymatisch & wenig oder fehlend \\
\hline
\end{tabular}

Tabelle 1: Untersuchungsergebnisse von jeweils 25 Nadelblättern je Art. 


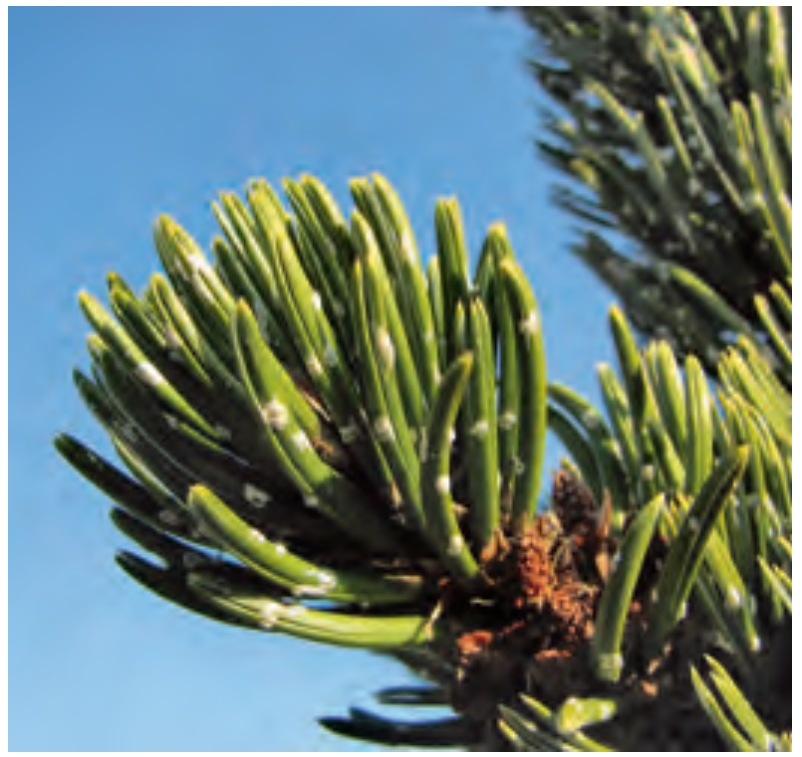

Abb. 10: Nadeln von Pinus aristata mit typischen Harzflocken. (Foto: V. M. DörkeN)

tionsperiode sehr kurz und beschränkt sich lediglich auf 2 Monate im Jahr, in denen das gesamte Jahreswachstum erfolgen muss (Thiel 1989). Auf der Südseite der Gebirge steigt die Vegetation deutlich höher im Vergleich zur Nordseite. In den Hochlagen des natürlichen Vorkommens dominiert $P$. aristata. In den mittleren Lagen tritt sie in Konkurrenz mit $P$. flexilis und Picea engelmannii. In tieferen Lagen wird sie von P. ponderosa, Quercus gambelii, Juniperus communis und Populus tremuloides bedrängt (WeIss 2012), denen sie in der Wüchsigkeit deutlich unterlegen ist. In den südlicheren Verbreitungsgebieten kommen vermehrt Abies lasiocarpa var. arizonica, Abies concolor, Pinus engelmannii, P. flexilis,. P. strobiformis und Picea pungens vor (WeIss 2012).

Pinus aristata ist ein meist mehrstämmiger Baum, der bis 15 (-18) m hoch wird. Die Stämme sind oft drehwüchsig. Im Gegensatz zu P. longaeva erreichen die Nadeln von $P$. aristata nur ein Alter von ca. 20 Jahren (Weiss 2012). Die Nadeln stehen zu fünft zusammen. Ausnahmsweise werden auch Kurztriebe mit 2-8 Nadeln gefunden (WeIss 2012). Die Nadeln werden $2-4 \mathrm{~cm}$ lang. Durch antrocknende Harztropfen werden die typischen auf fast jeder Nadel zu findenden weißen, wolllausartigen Harzflocken ausgebildet. Die beiden adaxialen Seiten der Nadel sind durch Stomatalinien auffällig weiß gefärbt, während die abaxiale
Seite leicht gefurcht und grün ist. Die Pollenzapfen sind überwiegend gelb, wobei gelegentlich auch rötliche oder bläulich-violett gefärbte gefunden werden (FARJON 2010). Diese abweichende Färbung könnte auf die enge Verwandtschaft zu P. longaeva hinweisen, deren Pollenzapfen rötlich gefärbt sind. Die Samenzapfen sind zum Zeitpunkt der Bestäubung purpurfarben und stark harzig. Die reifen Samenzapfen sind später graubraun gefärbt und ebenfalls noch stark harzig. Sie werden 6-11 cm lang und 5-8 cm breit. Auf dem Rücken der Samenschuppen ist der Schuppenschild (Apophyse) rhombisch bis dreieckig ausgestaltet. Dieser sitzt zentral eine 3-8 mm lange, abstehende Grannen auf. Die Zapfen reifen im September bis Oktober des folgenden Jahres. Die Samen von $P$. aristata sind wesentlich schwerer und dunkler als die von $P$. longaeva. Sie werden 5-6 mm lang. Der Samenflügel entspricht mit 7-15 mm Länge dem von $P$. longaeva.

Auch $P$. aristata kann ein beachtliches Alter erreichen. So wurde bei einem noch lebenden Exemplar ein Alter von 2435 Jahren nachgewiesen (WeIss 2012).

\section{Literatur}

Bailey, D. K. 1970: Phytogeography and taxonomy of Pinus subsection Balfourianae. - Ann. Missouri Bot. Gard. 57: 210-249.

Critchfield, W. B. 1977: Hybridization of foxtail \& bristolcone pines. - Madrońo 24: 193-212.

Eckenwalder ,J. E. 2009: Conifers of the world. - Portland, London.

FARJON, A. 2010: A handbook of the world's conifers. Vol. 1.Leiden, Boston.

Flanary, B. E \& Kletetschka, G. 2005: Analysis of telomere length and telomerase activity in tree species of various life-spans, and with age in the bristlecone pine Pinus longaeva. Biogerontology 6: 101-111.

Mirov, N. T. 1967: The genus Pinus. - New York.

Schütt P. \& Lang U. M. 2004a: Pinus balfouriana Grev. \& Balf., 1853. In: Schütt, P., Weisgerber, H., Schuck, H. J., Lang ,U. M., Stimm, B. \& Roloff, A. (Hrsg): Lexikon der Nadelbäume. - Hamburg. 


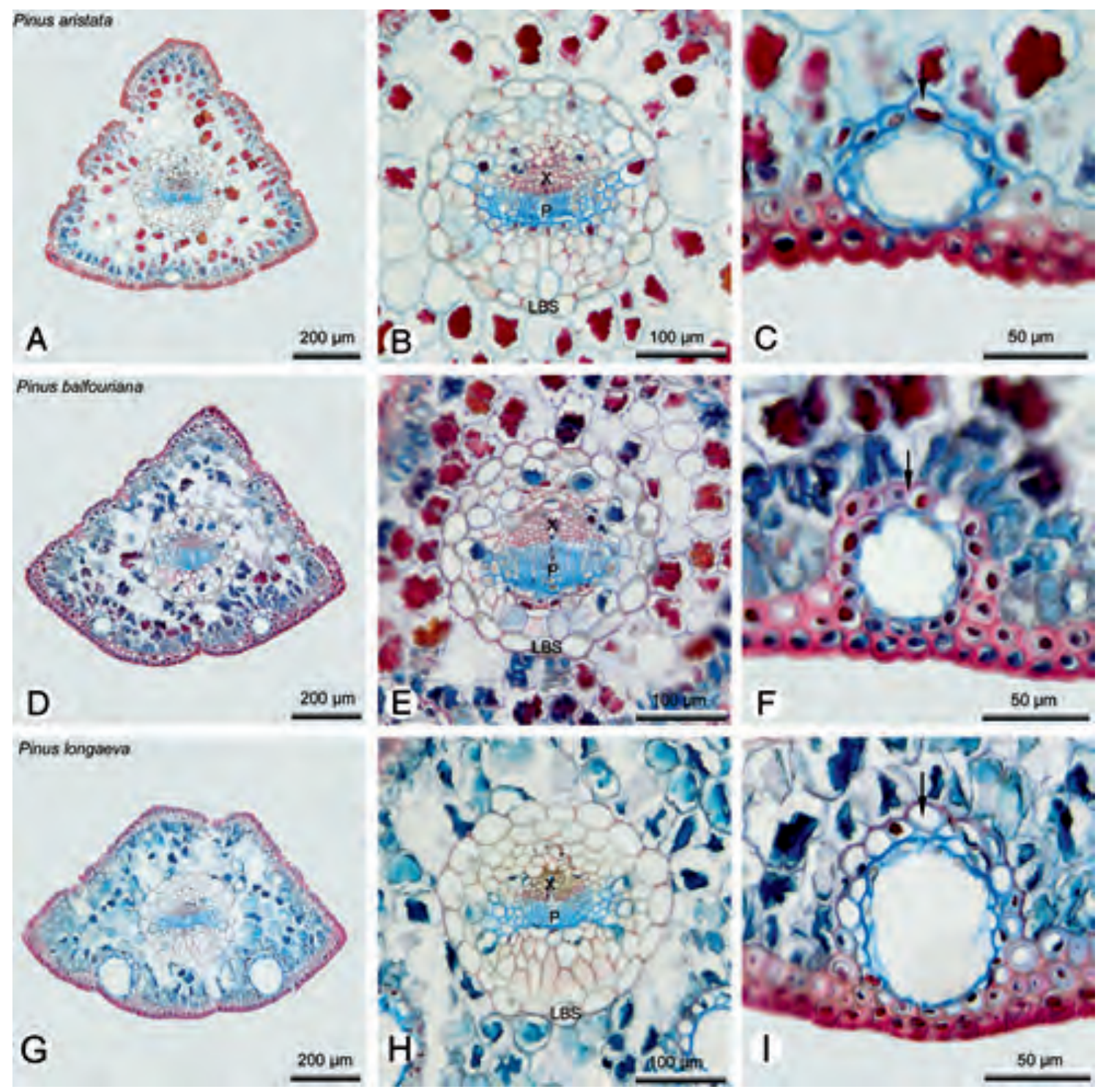

Abb.11: Mikroskopische Ansichten von Nadelquerschnitten der drei verschiedenen Foxtail Pines. (Foto: V. M. Dörken) A, D, G: Nadelblattquerschnitt; B, E, H: Leitbündeldetail; alle drei Arten mit deutlicher parenchymatischer Leitbündelscheide (LBS), Xylem (X) zeigt zur Blattoberseite, Phloem (P) zur Blattunterseite; C, F, I: Detail Harzkanal; Harzkanalscheide (Pfeil) bei Pinus aristata (C) dünnwandig und parenchymatisch, bei Pinus balfouriana (F) und Pinus longaeva (I) dickwandig und sklerenchymatisch.

Schütt, P. \& Lang, U. M. 2004b: Pinus longaeva D. K. BaILey 1970. In: Schütt, P., Weisgerber, H., Schuck H. J., Lang U. M., Stimm, B. \& Roloff, A. (Hrsg.): Lexikon der Nadelbäume. - Hamburg.

Thiel, W. 1990: Bei den ältesten Bäumen der Erde, den Grannenkiefern. - Mitt. Dtsch. Dendrol. Ges. 79: 149-157.

Weiss, H., 2012: Pinus aristata Engelman, 1862. In: Enzyklopädie der Holzgewächse 46. - Weinheim.

ZaVArin, E. \& SNAJBERK K. 1973: Variability of the wood monoterpenoids from Pinus aristata. - Biochem. Syst. Ecol. 1: 39-44.

Zavarin, E., Snajberk K. \& Bailey, D. 1976: Variability in the essential oils of wood and foliage of Pinus aristata and Pinus longaeva. - Biochem. Syst. Ecol. 4: 81-92.
Zavarin E., Snajberk K., Bailey, D. \& Rockwell, E. C. 1982: Variability in essential oils and needle resin canals of Pinus longaeva from eastern California and western Nevada in relation to other members of subsection Balfourianae. - Biochem. Syst. Ecol. 10: 11-20.

\section{Anschriften der Autoren}

Hubertus Nimsch, St. Ulrich 31, 79283 Bollschweil, E-Mail: hubertus.nimsch@t-online.de

Dr. Veit Martin Dörken, Universität Konstanz, Fachbereich Biologie M 613, Universitätsstraße 10, 78457 Konstanz, E-Mail: veit.doerken@uni-konstanz.de 